\title{
All-dielectric multiple quantum well active metasurfaces
}

\author{
Meir Grajower ${ }^{1}$, Junghyun Park ${ }^{2}$, Ruzan Sokhoyan ${ }^{1}$, Pin Chieh Wu ${ }^{1,3}$, Ghazaleh Kafaie Shirmanesh ${ }^{1}$, \\ Souvik Biswas ${ }^{1}$ and Harry A. Atwater ${ }^{1,4}$ \\ ${ }^{I}$ Thomas J. Watson Laboratory of Applied Physics, California Institute of Technology, Pasadena, CA 91125, USA. \\ ${ }^{2}$ Samsung Advanced Institute of Technology, Suwon, Gyeonggi-do 443-803, South Korea \\ ${ }^{3}$ Department of Photonics, National Cheng Kung University, Tainan 70101, Taiwan. \\ ${ }^{4}$ Kavli Nanoscience Institute, California Institute of Technology, Pasadena, CA 91125, USA. \\ Author e-mail address: meirg@,caletch.edu
}

\begin{abstract}
We report a design of an all-dielectric Mie-resonant active metasurface, which exhibits a high reflectance $(>45 \%)$ and a broad tunable phase shift at an operation wavelength of $1550 \mathrm{~nm}$.

The proposed design can be used for the realization of two-dimensional active metasurfaces. $\subseteq 2020$ The Author(s)
\end{abstract}

Metasurfaces have attracted tremendous attention due to their ability comprehensively control the wavefront of the scattered light at a subwavelength scale [1]. Metasurfaces can potentially replace conventional thick and bulky (at least, millimeter-scale) optical components with low profile sub-micron-thick structures. In addition, a single metasurface can exhibit functions which otherwise would only be achieved by combining multiple bulk optical components. For example, metasurfaces have been used to demonstrate polarization dependent lenses and vortex beam waveplates [1].

Most metasurfaces demonstrated so far do not allow post-fabrication tunability, but during the several last years, there has been a significant effort towards the development of actively tunable metasurfaces [2]. To realize metasurface-based spatial light modulators it is necessary to actively and locally control the phase of the scattered light (from $0^{\circ}$ to $360^{\circ}$ ) in a reversible fashion. The spatial phase control should be realized on a subwavelength scale while maintaining uniformly high reflectance. So far, active metasurfaces have been realized via a number of different approaches, which commonly rely on incorporating an active material into the metasurface structure. The dielectric permittivity of the active material is then dynamically controlled via application of an external stimulus, such as an electrical bias, laser pulse, or heat input [1]. Recently, a universal active metasurface based on a plasmonic cavity has been demonstrated. The demonstrated metasurface has been able to perform multiple different optical functions depending on the bias application configuration. Nonetheless, the reflectance of the metasurface is relatively low $(<20 \%)$ [3], limiting its overall power efficiency.

Here, we develop a design of an all-dielectric metasurface, which exhibits both high reflectance $(>45 \%)$ and an actively tunable phase shift of $300^{\circ}$ at a wavelength of $1550 \mathrm{~nm}$. The proposed metasurface consists of an array of dielectric Mie resonator pillars made of InGaAs/InAlAs multiple quantum wells (MQWs) [4]. When a direct current (DC) electric field is applied across the quantum wells, the refractive index of the MQWs is modulated due to the quantum-confined Stark effect (QCSE) [5]. In our case, the QCSE enables relatively large modulation of the complex refractive index of the MQW layer $(\Delta \mathrm{n} \sim 0.02)$ at wavelengths near the MQW bandgap. In the proposed metasurface design, the dielectric resonators are encapsulated between a high refractive index doped Si substrate and a top Au mirror (Fig. 1a). The Au mirror and the doped Si layer are used as electrodes, enabling the application of the DC electric field across the MQW pillars. The amplitude and phase of the light reflected from the metasurface can be actively controlled by applying voltage. In our prior work, we have demonstrated all-dielectric active metasurfaces based on electro-optically tunable GaAs/AlGaAs MQWs patterned into subwavelength elements that each supports a hybrid Mie-guided mode resonance [6]. While we have been able to observe a relative reflectance modulation of $270 \%$, the observed tunable phase shift has been limited to $70^{\circ}$. Our proposed metasurface design overcomes the limitations encountered in our previous work [6] as a result of the improved properties of InGaAs/InAlAs MQWs, as well as the modified metasurface design.

The metasurface antenna element performance was simulated using finite difference time domain (FDTD) calculations. In our simulations, we assume a silicon substrate of a finite thickness of $330 \mathrm{~nm}$. Our simulations show the metasurface exhibits a continuous phase shift of the reflected light of $300^{\circ}$ upon application of a bias voltage. The Au mirror enables a high reflectance, which in our case is above $80 \%$ (inside the silicon). Since the observed phase shift is not due to a long-range coupling between resonators, our tunable metasurface element is compact and thus compatible with full two-dimensional (2D) control of the wavefront of the reflected light at a subwavelength scale.

We have also analyzed the metasurface beam steering performance. Figure $1 \mathrm{~b}$ exhibits the far-field radiation intensity in the case when the phase profile of the active metasurface corresponds to one of the binary phase grating 
[see the left inset of Fig. 1b)]. The binary grating phase profile can be achieved via an appropriate bias application. The supercell of our binary grating consists of 8 Mie resonators. Within a grating period, 4 adjacent Mie resonators provide a phase shift of $0^{\circ}$ while the other 4 Mie resonators provide a phase shift of $180^{\circ}$. In our simulations, the period of our binary phase grating is $5.2 \mu \mathrm{m}$. When no electrical bias is applied the incoming light is reflected back to the $0^{\text {th }}$ diffraction order. When we apply voltage in the binary phase grating configuration, the light is efficiently steered to $\pm 1^{\text {st }}$ diffraction orders [Fig. 1b)].
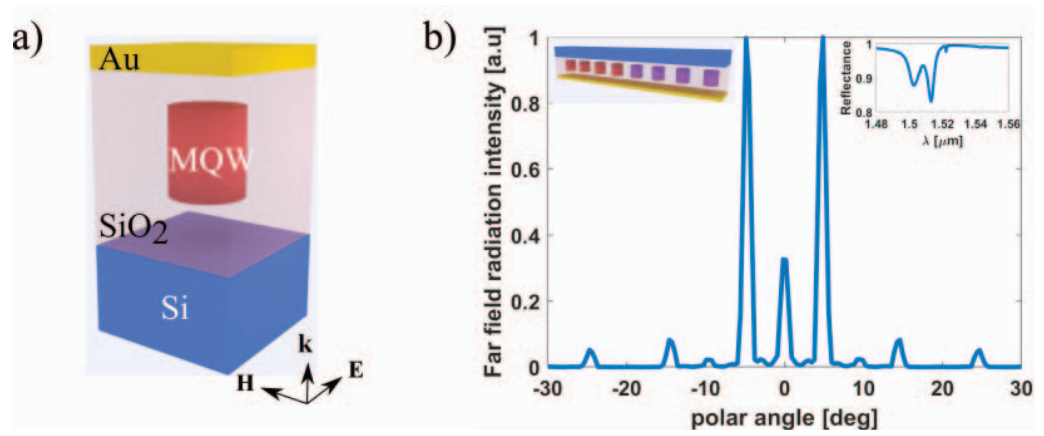

Figure 1 - a) A unit cell of a MQW metasurface. The unit cell consists of a Si substrate, a cylindrical Mie resonator made of MQW. The resonator is encapsulated inside a silica layer. On top of the oxide, we deposit a metallic mirror. The top mirror and the Si layers act as electrodes. The period of the metasurface is $650 \mathrm{~nm}$. The pillar radius is $280 \mathrm{~nm}$, and the pillar height is $500 \mathrm{~nm}$. b) Beam steering by a Mie-resonant metasurface. In the right inset, the reflectance spectrum is presented. A high reflectance $(>80 \%)$ is maintained over the considered spectral region. The left inset is a schematic of the simulated binary grating.

As noted above, the reflected light is recorded inside the silicon layer. As such, the calculated polar angle is inside the silicon substrate. Consequently, the propagation angle in free space would be $\theta=\operatorname{asin}\left(n_{s i} \sin \left(4.8^{\circ}\right)\right)=$ $17^{\circ}$ and the overall efficiency is $45 \%$. From the experimental point of view, the proposed metasurface design can be realized by bonding MQW structures onto a silicon on insulator (SOI) wafer, followed by etching of Mie resonator pillars and subsequent deposition of $\mathrm{SiO}_{2}$ and gold interconnects.

In conclusion, we introduce a new active metasurface design based on Mie-resonant MQW active elements [4]. Our design is based on local Mie resonators which enable 2D local control on each resonator, which is compatible with design of two-dimensionally architected metasurface. Simulations indicate the potential for active beam steering while maintaining high reflectivity $(>45 \%)$.

\section{References}

1. H. T. Chen, A. J. Taylor, and N. Yu, "A review of metasurfaces: Physics and applications," Reports Prog. Phys. 79, (2016).

2. M. C. Sherrott, P. W. Hon, K. T. Fountaine, J. C. Garcia, S. M. Ponti, V. W. Brar, L. A. Sweatlock, and H. A. Atwater, "Experimental Demonstration of $>230^{\circ}$ Phase Modulation in Gate-Tunable Graphene-Gold Reconfigurable Mid-Infrared Metasurfaces," Nano Lett. 17, 3027-3034 (2017).

3. G. K. Shirmanesh, R. Sokhoyan, P. C. Wu, and H. A. Atwater, "Electro-Optically Tunable Universal Metasurfaces," arXiv:1910.02069 1-10 (n.d.).

4. Z. Xu, C. Wang, W. Qi, and Z. Yuan, "Electro-optical effects in strain-compensated InGaAs/InAlAs coupled quantum wells with modified potential," Opt. Lett. 35, 736 (2010).

5. D. A. B. Miller, D. S. Chemla, T. C. Damen, A. C. Gossard, W. Wiegmann, T. H. Wood, and C. A. Burrus, "Electric field dependence of optical absorption near the band gap of quantum-well structures," Phys. Rev. B 32, 1043-1060 (1985).

6. P. C. Wu, R. A. Pala, G. Kafaie Shirmanesh, W. H. Cheng, R. Sokhoyan, M. Grajower, M. Z. Alam, D. Lee, and H. A. Atwater, "Dynamic beam steering with all-dielectric electro-optic III-V multiple-quantum-well metasurfaces," Nat. Commun. 10, 3654 (2019). 